\title{
Research on the Three Dimensional Modeling Method of Automobile Steering Rocker Shaft Non-Circular Sector Gear
}

\author{
Jin-Fang YU a, Guo-Long DING, Ya-Li ZHANG \\ School of Mechanical Engineering, Hubei University of Technology, Wuhan 430068 \\ 1038430308@qq.com
}

\begin{abstract}
Keywords: Automobile steering, Rocker shaft, Non-circular sector gear, Three dimensional modeling.
\end{abstract}

\begin{abstract}
The precise 3D(Three Dimensional) modeling of the non-circular sector gear of the rocker shaft is an important basic work for the study of the mechanical properties of the automobile steering. The traditional 3D modeling method is difficult to obtain accurate model of the rocker shaft because of its special gear surface structure. Through the analysis of the meshing movement rules and the numerical control machining process of the non-circular sector gear, a kind of 3D modeling method based on the actual gear shaping process of the non-circular sector gear is proposed. Combined with instance data, the two-dimensional simulation model in MATLAB was firstly carried out, and we also found out that the tooth profile of the big end face and small end face of the non-circle sector gear is the offset curve rather than the shift curve. The accurate 3D model of the non- circular tooth fan was built by Solidworks, and after leading-in the model to ANSYS, it was found that the model could not be meshed. Finally, the model dressing method was proposed, and the 3D model which can be meshed is obtained through the method of segment the gear workpiece by extracting the envelope surface of the cutter motion.
\end{abstract}

\section{Introduction}

The rocker shaft non-circular sector gear is a very important part in the recirculating ball steering system, which has an important influence on the steering performance of the steering. When analyzing the static or dynamic analysis of automobile steering gear, we usually requires the use of ANSYS and other software to analysis the mechanical property of 3D model of steering gear, So the precision of the steering gear modeling plays an important role in the accuracy of the simulation analysis. The ratio of the rocker shaft non-circular gear couple is usually designed as a variable transmission ratio[1,2], so the general modeling method provided by the three dimensional software cannot model accurately. It's usually requires the use of computing software (such as MATLAB) to calculate the tooth profile coordinates value, and then import it into the three-dimensional software to model. This method is very effective for the modeling of cylindrical spur gears, but it is unable to model the rocker shaft with variable thickness tooth[3,4] accurately.

Many scholars have put forward different methods in the modeling of the rocker shaft non-circle sector gear. ZENG[5] carried out the 3D modeling of the eccentric rocker shaft non-circular sector gear which provides the basis for the following analysis of meshing transmission. But the tooth profile curve of end section of non-circular gear with variable thickness tooth is the offset curve rather than the shift curve, so the method used in this paper is not to establish a precise 3D model. ZHANG[6] uses CAXA to compute the large end tooth profile and the small end tooth profile of the rocker shaft, and then imported it into UG to generate the gear's 3D model. This method can only be used to modeling the rocker shaft which has a fixed transmission ratio. For the non-circular tooth profile coordinates which has a variable transmission ratio, the CAXA cannot work out. XIE[7] and HU[8] uses the idea of simulation process, which has the characteristics of high accuracy of modeling, but there is no study on the modeling of the non-circular sector gear with variable thickness tooth. Based on the virtual machining principle, the workpiece is fixed and the cutting tool is rotated and translated, and the 3D model of the generated surface gear is accurate and reliable[9]. The above modeling methods don't take into account such problems: whether the mechanical analysis of the 3D model can be further carried out. The finite element method is 
commonly used in the mechanical analysis, and the key of the finite element method is the mesh of entities. Therefore, the 3D modeling of the rocker shaft non-circular sector gear is carried out on the premise of the model can be meshed.

In this paper, the 3D model of the rocker shaft non-circular sector gear was carried out in the Solidworks, which was based on its NC machining technology[10]. Solid Boolean subtraction was mainly used when building a model, which is similar to the actual processing of non-circular sector gear. At the same times, although the model which was modeled by this method has a high precision, but when imported this model into ANSYS to mesh, it will be warned that there are too many segments and some of them are too short, which lead the mesh could operate to fail. Therefore, this paper proposes a model modification scheme at the same time. By extracting the envelope surface which is formed by the movement of tool, and then use this curved surface to segment the workpiece model of sector gear to obtain a relatively accurate 3D model which can be mesh in ANSYS.

\section{Processing Methods of Steering Rocker Shaft Non-Circular Sector Gear}

Nowadays, the design method of recirculating ball steering non-circular sector gear is the variable thickness tooth one, not only has the advantages of convenience to the assembly of rocker shaft gear rack, and can ensure that the rocker shaft gear sector can be closed contact with the meshed rack, making steering non-circular rack-and-pinion to achieve non-backlash when they are matched. Non backlash meshing of the non-circular rack-and-pinion will reduce the vibration of the steering wheel caused by the vibration of the vehicle, and increase the safety and stability of the vehicle.

\section{Meshing Transmissions Analysis}

The recirculating ball steering gear consists of two levels of speed changing mechanism, which is a screw nut pair and a rack and pinion gear pair, using $i_{1}$, $i_{2}$ to represent its transmission ratio, so the total transmission ratio $i$ can be represent by $i_{1}, i_{2}$, thus:

$$
i=i_{1} \cdot i_{2}
$$

where $i_{1}=2 \pi / p, \quad p$ represents the helical pitch of the screw nut pair .

So the ration of the sector gear, $i_{2}$ can be expressed by:

$$
i_{2}=p i / 2 \pi=r
$$

Where $r$ is defined as the instantaneous radius of the sector gear.

The moving distance of the rack is relate to the angle of the sector gear, thus:

$$
l(\theta)=\int_{0}^{\theta} i_{2} \mathrm{~d} \varphi
$$

Mesh point is the intersection of the normal line and the common tangent of the tooth profile, so the point can be determined by solving the equations simultaneously. Figure 1 shows the coordinate system of solving the mesh point. The common tangent equation of the mesh point is the tooth profile of the cutter, which is involved in the mesh. The tooth profile of the cutter is a straight line segment, then the equation of the common tangent can be found by:

$$
y-y_{0}=\tan \left(\alpha_{1}+\pi / 2\right)\left(x-x_{0}\right)
$$

where $\left(x_{0}, y_{0}\right)$ is a known coordinate point of the profile on the rack which is involved in the mesh; $\alpha_{1}$ is the pressure angle of the profile on the rack which is involved in the mesh.

According to the Willis' theorem, it can be realized that instantaneous center lies in the normal line, and the normal line is perpendicular to the common tangent, so the equation of the common tangent can be defined as:

$$
y-r=\tan \left(\alpha_{1}\right) x
$$

The coordinate of the mesh point within the fixed coordinate system can be solved by the 
simultaneous equations, namely:

$$
\left\{\begin{array}{l}
y-y_{0}=-\cot \left(\alpha_{1}\right)\left(x-x_{0}\right) \\
y-r=\tan \left(\alpha_{1}\right) x
\end{array}\right.
$$

The formula above is the trajectory equation of the mesh point within fixed coordinate system.

The rotation formula is:

$$
\left\{\begin{array}{l}
x_{1}=x \cos (\theta)-y \sin (\theta) \\
y_{1}=x \sin (\theta)+y \cos (\theta)
\end{array}\right.
$$

Where $\theta$ is defined as the rotation angle of gear when the mesh point is meshing.

The coordinate of the mesh point within the coordinate system of gear can be obtained by the way of rotating the mesh point the angle of the gear in the opposite direction around the fixed coordinate system. Formula.7 represent the equation of the flank profile, where $\left(x_{1}, y_{1}\right)$ is the coordinate of any point in the profile.

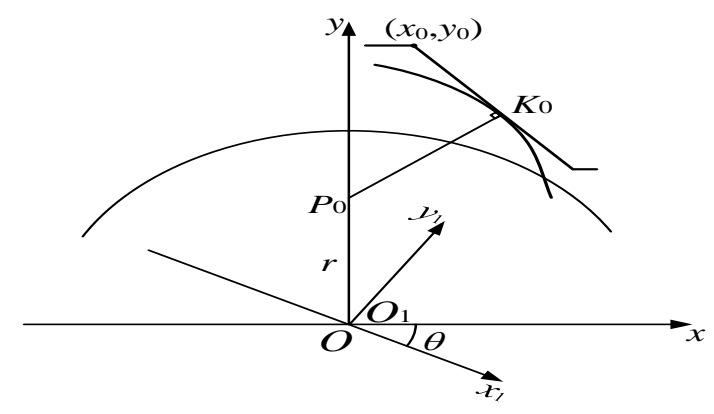

Figure 1. Solution illustrative diagram of the flank profile' coordinate

\section{CNC Machining Methods}

Automobile steering rocker shaft non-circular gear usually is an eccentric one, which is shown in Figure 2, that is to say, the gyration center of the rocker shaft is not in the geometry center of the non-circular sector gear. The design of the eccentric sector gear is to adjust the back lash of the non-circular sector gear and rack pair with different rotation angle.

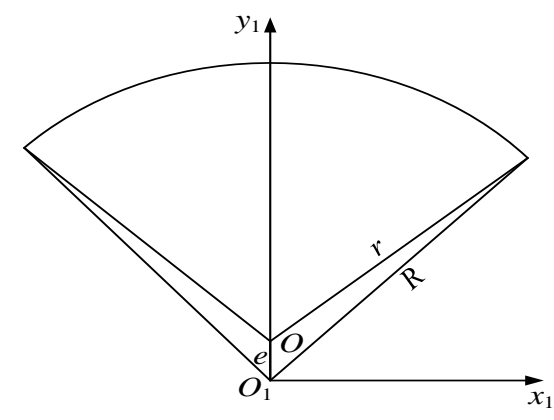

Figure 2. Illustrative diagram of the eccentric gear

Automobile steering rocker shaft non-circular sector gear is often made by gear shaping. As to the rocker shaft non-circular sector gear with variable transmission ratio, it can only be processed by the gear shaper. The main movement of the gear shaping is the up and down stroke movement of the tool generally, and the feed movement consist of three groups: the front-back feed motion of the radial feed shaft (Y-axis), the left-right feed of the horizontal feed shaft (X-axis) and the rotary motion of the rotating shaft $(\mathrm{C}$-axis) of the work piece, where $\mathrm{X}$-axis and $\mathrm{C}$-axis process the generating motion based on the given transmission gears ratio curves, as shown in Figure 3. 


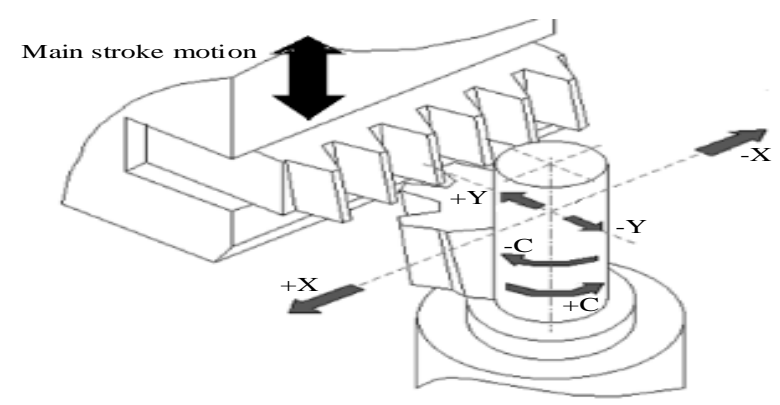

Figure 3. Illustrative diagram of the profile modification of gear cutting and shaping process

The eccentric non-circular sector gear of the automobile steering rocker shaft is a sector gear shaft. It can be cut by using the eccentric fixture. Nowadays, because it is difficult to manufacture the eccentric fixture, and to guarantee the installation accuracy, moreover, an eccentric fixture can only process one type of parts. So we can develop the special numerical control system to control the linkage interpolation of each axis accurately rather than using the eccentric fixture. The relation between the axis movement and the angle of the gear is as follows:

$$
\left\{\begin{array}{l}
x(\theta)=e \cos \theta \\
y(\theta)=R+e \cos \theta \\
c(\theta)=\theta
\end{array}\right.
$$

The interpolation motion relation which is shown in Formula. 8 can ensure the process of the eccentric non-circular sector gear.

\section{Modeling Example of 3D}

The example comes from one product of a steering gear manufacturer. The ratio curve is made up of several line segments, and the rocker shaft sector gear has eccentricities in a certain range. According to the theory above, the sectional line transmission ratio is adopted to process the non-circular sector gear (Figure 4) on a CNC gear shaping machine. The main parameters of this example is shown in Table.1, and the shape of the used tool is shown in Figure 5.

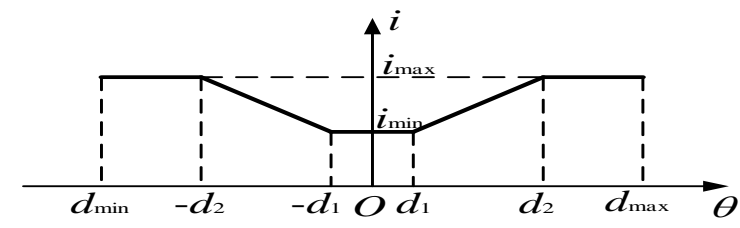

Figure 4. Sectional line transmission ratio

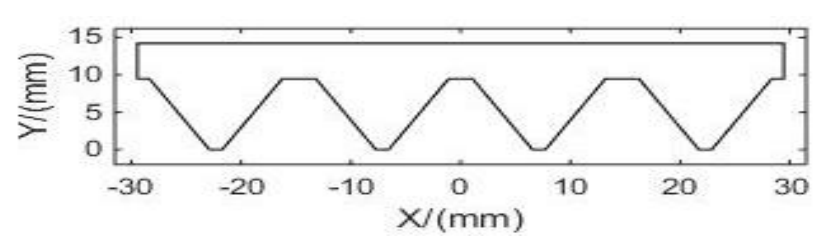

Figure 5. Shape of the used tool

Table 1. Main parameters of the sector gear

\begin{tabular}{|l|l|}
\hline Parameter & value \\
\hline Big end diameter $D_{\max } /(\mathrm{mm})$ & 85 \\
\hline Total cutting amount $\mathrm{ap} /(\mathrm{mm})$ & 7.58 \\
\hline Minimum transmission ratio angle of termination $d_{1} /\left(^{\circ}\right)$ & 4 \\
\hline Maximum transmission ratio angle of termination $d_{2} /\left(^{\circ}\right)$ & 22 \\
\hline Minimum transmission ratio $I_{\min }$ & 24.0 \\
\hline Maximum transmission ratio $I_{\max }$ & 26.329 \\
\hline Steering gear screw pitch $L /(\mathrm{mm})$ & 9.525 \\
\hline Maximum eccentricity $e_{m} /(\mathrm{mm})$ & 0.2 \\
\hline Cutting angle $\gamma /\left(^{\circ}\right)$ & 2 \\
\hline Sector gear angle $\theta /\left(^{\circ}\right)$ & 90 \\
\hline
\end{tabular}




\section{D Modeling of MATLAB}

When using the gear shaper to process the automobile steering rocker shaft non-circular sector gear, it only need to control the linkage interpolation relation between the axis of the machine on the basis of the presetting transmission ratio function and variable displacement function. Simulating the linkage interpolation relation between the axis of the machine and the process of the non-circular sector gear in MATLAB before the actual machining process and then using the simulation results can assess the correctness and accuracy of the presetting laws of the machine axes. Taking the large end face and small end face as an example, the simulation results of the eccentric non-circular sector gear are shown in Figure 6.

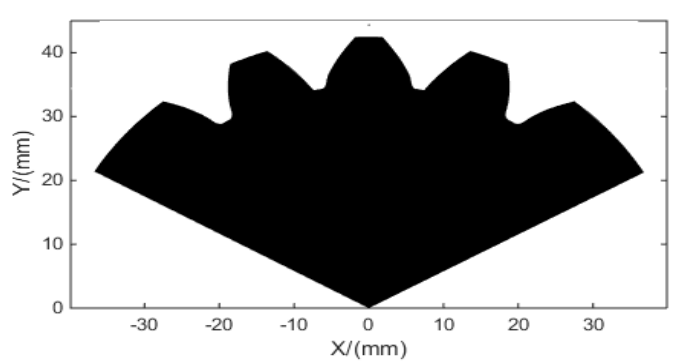

a. Big end face tooth profile

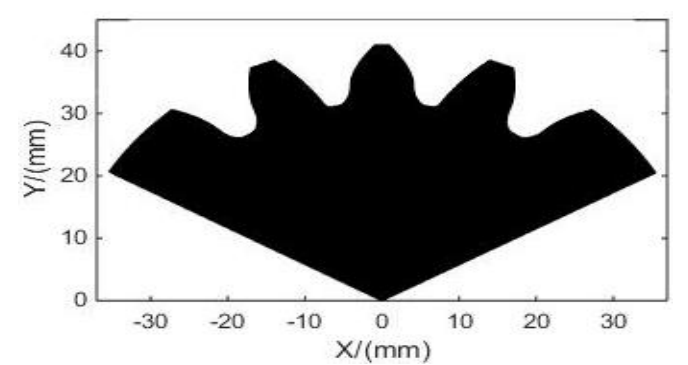

b. Small end face tooth profile

Figure 6. Tooth profile shape of the eccentric non-circular sector gear

As shown in the simulation graphics, it is clear that the tooth profile shape of the big end face differ from that of the small obviously. There is no undercutting at the root of the big end face, while the small end face has the over cut.

\section{D Modeling of Solidworks}

Because the steering rocker shaft non-circular sector gear has variable tooth thickness, their is an angle between the principal axis of the gear shaping machine and the $\mathrm{Z}$-axis of the machine tool coordinate system which is named as cutting angle. Due to the cutting angle, the tooth profile shape of the big end face is different from that of the small end face. According to the analysis of the meshing motion of the non-circular gear, it is known that the tooth curve(without considering the tooth profile at the undercutting part) along the axial section of the the non-circular sector gear is equidistant curve, so that it is impossible to use the stretch excision method to model its 3D modeling. What's more, the undercutting of the non-circular sector gear results in the difficulty of accurate 3D modeling.

Base on the NC machining technology of the rocker shaft non-circular sector gear, the 3D modeling method of virtual machining is proposed in this paper. This method is to use the entity Boolean operation provided by Solidworks to achieve the 3D model of the non-circular sector gear. There are the steps as follows:

(1) First, using the two development programming function of Solidworks to work out the space position of the rack in the different angle of the gear in bulk, as shown in Figure 7.

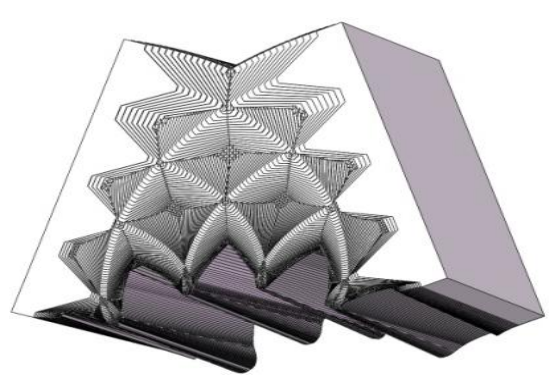

a. Big end face

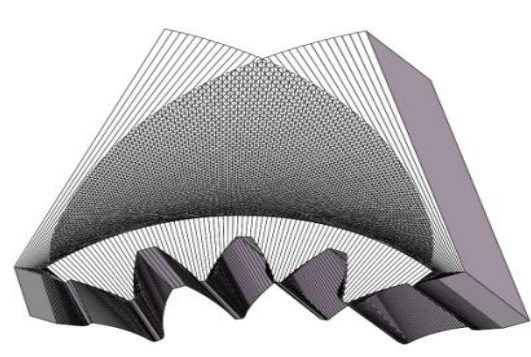

b. Small end face

Figure 7. Superposition of the rack in different position 
By using the Boolean addition operation to pull together the rack in different position, the final geometry is defined as the sweeping area in space of the rock tool in the process of the NC machining of non-circular gear.

(2) Second, setting a 3D model of the roughcast of the gear. In order to make it easy to set the model, using a tapered sector boss to represent the rough. Then, using the Boolean operation of Solidworks to make the rough as the main body, and make the tool assembly model as the minus body, which can result in the accurate model of the non-circular sector gear, as shown in Figure 8 and Figure 9.

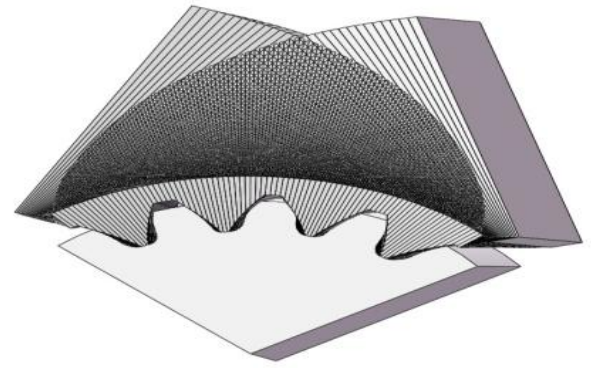

Figure 8. Inserting the rough of the gear

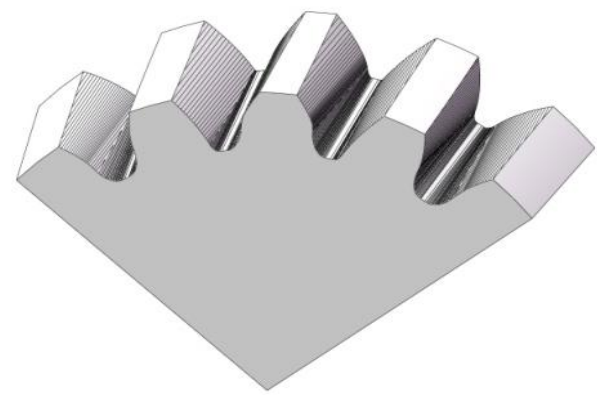

Figure 9. 3D model of the gear

With this, the 3D model of the sector gear has been finished. However, it goes wrong when importing this model into ANSYS to do the mesh generation. ANSYS put forward a tip that some segments of the model are too short to make the mesh. The reason for the mistake is that the 3D model method which is based on the actual processing of the gear cause a great deal of segments on the tooth surface of the gear 3D model, the cause of which is the same as that of the tooth marks in the actual gear process.

\section{Improvement of Model}

The 3D model was generated by using the above method can not be meshed, so the static and dynamic analysis of the non-circular sector gear rack pair can not be carried out by using ANSYS.

The reason is that there are too many line segments on tooth surface and the some of them are too short. Therefore, it is necessary to use a processing method to fit the curve on the tooth surface, and then use that surface to replace a series of plane on the 3D model which is generated by the front method (By analyzing the model's formation mechanism, it is known that the tooth surface of the model is composed of a series of plane). Specific improvement methods are as follows:

(1) Tool assembly is cut by two plane, as shown in Fig. 10 that is in order to extract the envelope curve of the tool's movement. The envelope curve was extracted in the two end of the tool assembly after cutting (by using the curve function of through the reference point in Solidworks), and then use the two envelope curve to construct the boundary surface, which is the envelope surface of the motion of the rack cutter, as shown in Figure 11:

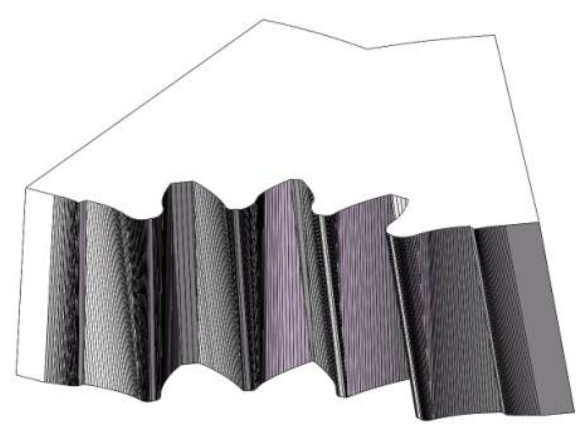

Figure 10. Tools assembly after repaired

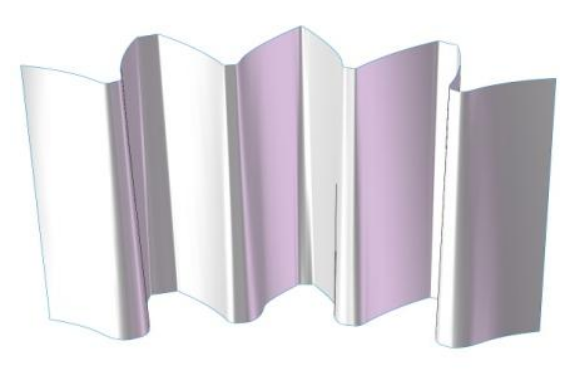

Figure 11. Envelope surface of the motion of the rack cutter 
(2) As shown in Figure 12, we set up a 3D model of the sector gear workpiece, and segment it by the envelope surface of the motion of the rack cutter, and then the 3D model of the non-circular sector gear is obtained, whose tooth surface is curved surface. And then imported the model into ANSYS to mesh, the grid is shown in Figure 13.

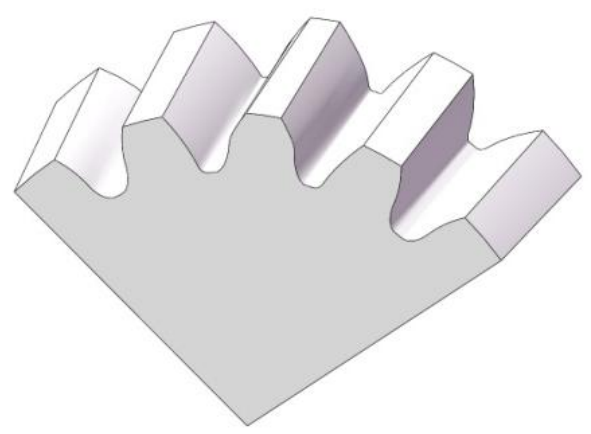

Figure 12. Sector gear profile model with curved surface

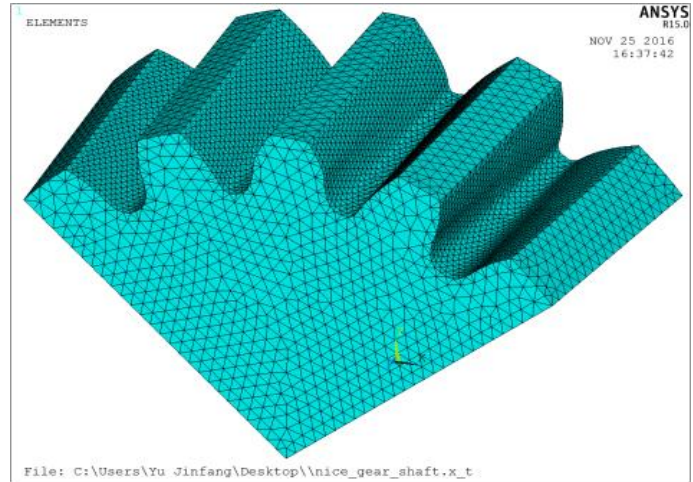

Figure 13. Sector gear meshing

\section{Conclusion}

(1) The tooth profile shape of the big end face and small end face of the automobile steering rocker shaft non-circle sector gear is different. The profile curves of the different cross section is the offset curve rather than the shift curve, so we can not use the method of stretching to model the rocker shaft non-circular sector gear.

(2) Based on the 3D modeling method of the NC machining process of the rocker shaft non-circular sector gear, the 3D model of the rocker shaft with high precision can be generated. However, the tooth profile of the model is composed of many small planar surfaces, so there are too much lines on the tooth profile surface, leading to the model imported into the ANSYS can not be meshed, which hinders the further analysis of the static and dynamic analysis of the steering non-circular sector gear rack pair.

(3) After using the envelope curve to construct the envelope surface, and then use that surface to segment the sector gear workpiece model, the profile surface of the tooth profile is changed into several curved surfaces, rather than the plane combination, so, importing the improved 3D model of the sector gear into the ANSYS can be a good mesh, which shows that the improved method has good practicability. Because the envelope surface is extracted according to the position of the cutting tool's movement, the improved method also has high accuracy.

\section{Acknowledgment}

The authors would like to thank the Science and Technology Hall of Hubei, China for financially supporting the part of this work.

\section{References}

1. HE J L, QIN J X, WU X T. A Study on Design Theory of Sector Steering Gear with Variable Ratio [J]. Journal of Mechanical Transmission, 2009, 33(1):12-14.

2. DUAN B. Variable Ratio Rack and Pinion Steering Design and Finite Element Analysis Research. Wuhan University of Technology, 2013.

3. ZHANG F N, XIAO D Y, SHI H. A Study to a Thickened-tooth of a Motor Steering Gear [J]. Drive System Technique, 2001(4):14-19.

4. ZHANG F N. Eccentric Tooth On The Impact of Contact Ratio[J]. Drive System Technique, 2011, 25(4):12-14.

5. ZENG C H, LIAO H P. Precise 3D Modeling of Design of Arm Shaft. Development and 
Innovation of Machinery and Electrical Products, 2006, 19(4):163-163.

6. ZHANG L W, LIU Y G, ZHANG J F, et al. The Accurate Modeling of the Modified Sector Gear in NX6.0 by Using CAXA [J]. Digital Manufacturing Industry, 2012(12):45-47.

7. XIE G, YIN G F, ZHOU D C. 3D Dynamic Simulation Design of Automobile Steering Gear Variable Ratio - Gear Tooth Profile [J]. Machine Tool and Hydraulics, 2003(4):133-134.

8. HU D W, NIU Z R, TU M, et al. Digital Design Method of Variable Ratio Tooth Profile of the Recirculating Ball Variable Ratio Steering [J]. Journal of Mechanical Transmission, 2016(1):87-90.

9. LIN C, LI S S, GONG H. Design and 3D Modeling of Orthogonal Variable Transmission Ratio Face Gear [J]. Journal of Human University (National Sciences), 2014, 41(3):49-55.

10. DING G L, WANG P J. Numerical Control Machining of Eccentric Gear Sector for Steering Gear Rocker Arm Shaft [J]. Automobile Technology \& Material. 2009, No.5:56-58. 